\title{
Predictors of Breastfeeding Self-efficacy in Iranian Women: A Cross-Sectional Study
}

\author{
Mojgan Mirghafourvand ${ }^{1}$, Jamileh Malakouti², Sakineh Mohammad-Alizadeh-Charandabi ${ }^{3}$, Fatemeh \\ Faridvand $^{4^{*} \mathbb{D}}$
}

\begin{abstract}
Objectives: Determining the effective factors on breastfeeding self-efficacy can be helpful in designing the promoting interventions for this behavior. The aim of this study was to evaluate the predictors of breastfeeding self-efficacy in mothers referred to health centers of Tabriz, Iran, in 2015.

Materials and Methods: This cross-sectional study was conducted on 220 mothers breastfeeding 4-to-6-month babies. Cluster sampling was used in this study. Breastfeeding self-efficacy scale (BSES), The Iowa Infant Feeding Attitude Scale (IIFAS), social support via Personal Resource Questionnaire (PRQ-85) and researcher-made questionnaire of knowledge were used to collect data. Multivariate linear regression model was used to determine the predictors of breastfeeding self-efficacy.

Results: Mean (standard deviation) of breastfeeding self-efficacy was 138.7 (11.9) out of the achievable score range of 33-165 and about $90 \%$ of the women had high breastfeeding self-efficacy. There was a significant statistical correlation between breastfeeding self-efficacy and social support, knowledge and attitude $(P<0.001)$. According to multivariate linear regression model, the variables of social support, attitude, knowledge and husband's age were identified as the predictors of breastfeeding self-efficacy.

Conclusions: According to the important role of breastfeeding in maternal health, providing strategies to enhance knowledge, improve attitude, and sensitize community deems essential; as these important factors affect breastfeeding self-efficacy.

Keyword: Breastfeeding self-efficacy, Social support, Knowledge, Attitude, Iran
\end{abstract}

\section{Introduction}

Breast milk not only protects the health in childhood, but also provides human health throughout life (1). There is definite evidence about both short-term and longterm health benefits of breastfeeding for mothers and infants (2-8) and also its positive effects on increasing the binding and attachment between mother and infant and decreasing family costs. Avoiding breastfeeding is responsible for $45 \%$ of children mortality as the result of infection, $30 \%$ as the result of diarrhea, and $18 \%$ because of acute respiratory problems (9). Despite the supports of national and international organizations, most mothers stop breastfeeding before their infants achieve 6-12 months (10). Unfortunately, the prevalence and duration of exclusive breastfeeding have decreased in recent years (11). According to statistics, in 2010 in East Azerbaijan province, Iran, only $58.76 \%$ of newborns, in their first 6 months of life, have been exclusively breastfed by their mothers (12).

Self-efficacy is defined as an individual's beliefs in his capabilities in order to implement a definite way for achieving specific goals (1). Breastfeeding self-efficacy is originated from one of the structures of Bandora's socialcognitive theory (1977) and includes the individual's confidence and belief in his capabilities in implementing health behaviors such as successful exclusive breastfeeding. According to Dennis, there is a significant relationship between promoting mother's breastfeeding self-efficacy and the increase of breastfeeding duration (13). Breastfeeding self-efficacy depends on the mother's capability and self-confidence for breastfeeding and affects her decision for continuing it (14). It is one of the predictors of breastfeeding that shows mother's stability in continuing breastfeeding and her attempt to reach it $(15,16)$.

Several factors such as young maternal age, educational level, lower income, full-time working, smoking, C-section (17), postpartum depression, anxiety and breastfeeding problems like mastitis can reduce breastfeeding selfefficacy (18-20).

A study in Indonesia showed that self-efficacy affects breastfeeding (21). Moreover, another study on pregnant women above 18 years old, in their 37 weeks of pregnancy, showed that mothers with high self-efficacy had high exclusive breastfeeding in comparison with the mothers with low self-efficacy (1). According to the studies in Iran, unfortunately almost half of the Iranian mothers had low breastfeeding self-efficacy; as in the study by Varaei et al (1), $44 \%$ of mothers, and in the study by Rahmatnejad et al (22), $49 \%$ of mothers had low breastfeeding self-efficacy. However, the very low breastfeeding self-efficacy was 
also reported; as in the study carried out by Hasanpoor et al in Ahvaz city, Iran, only $2.5 \%$ of mothers had high breastfeeding self-efficacy. Factors such as the first pregnancy and lack of breastfeeding experience were mentioned as the reasons of low breastfeeding self-efficacy (23). Hence appropriate strategies are recommended for increasing the mother's breastfeeding self-efficacy (22). One of the possible solutions is giving information to the mothers and affecting their attitude about breastfeeding and increasing social support.

Social support means perceiving the availability of individuals who provide care for us (24). In a qualitative study by Barona-Vilar et al, the women with high social supports were more successful in breastfeeding (25). The results of a study conducted by Ericson et al on the effectiveness of proactive telephone support, provided for breastfeeding mothers of preterm infants, showed that the prevalence and duration of exclusive breastfeeding in the intervention group was more than that in the control group (26). Likewise, Nesbitt et al in their study showed that social and friendly relations, social support and mother's knowledge about the advantages of breastfeeding are some of the facilitating factors of breastfeeding (27).

Studies in Saudi Arabia and Mexico have referred to lack of correct information and knowledge, low awareness and false beliefs about insufficiency of breastfeeding for newborn's growth, as barriers against breastfeeding (28, 29). Giving information and training lead to success in breastfeeding through the change of behavior (30).

Sometimes, the main source of information is family and friends but their information is often insufficient. Supporting breastfeeding mothers and their knowledge are important factors in successful breastfeeding (31). Based on a study in Saudi Arabia, the main factor for unsuccessful breastfeeding is inconsistency in the knowledge and breastfeeding self-efficacy (32). Negative attitude of women, their husbands, and families is another barrier against breastfeeding (33); as a study in Saudi Arabia showed that attitude is a determinant factor in beginning and continuing breastfeeding (34).

Because of the importance of breastfeeding (4-6,8), decrease in exclusive breastfeeding (11), importance of breastfeeding self-efficacy in beginning and continuing it $(6,35)$, this study was conducted to determine the predictors of breastfeeding self-efficacy in the mothers referred to health centers of Tabriz, Iran.

\section{Materials and Methods}

Study Design and Participants

This cross-sectional study was conducted on 220 breastfeeding women referred to health centers of Tabriz city in 2015. The inclusion criteria were: being Iranian, willingness to participate in the study, ability to breastfeed, ability to read and write, having 4-6 months passed from delivery, and mothers of singleton term infant. Exclusion criteria included: women with stressful experiences within the past 6 months such as divorce, family disputes and loss of the beloved, those with unplanned pregnancy, existence of mental disorder in the mother and congenital anomalies in the newborn.

Regarding a previous study conducted on breastfeeding self-efficacy and by considering the precision of 0.05 around mean $(\mathrm{m}=101.7)$, confidence interval of $95 \%$ and statistical power of $90 \%$, and the largest standard deviation of 19.12 , which is related to breastfeeding self-efficacy scale (23), the sample size was determined about 110 cases and due to cluster sampling and considering the design effect $=2$, it was calculated as much as 220 cases.

\section{Sampling}

After obtaining necessary approval from the Ethics Committee of Tabriz University of Medical Sciences, a double-stage cluster sampling was conducted; as initially out of 39 and 42 health centers and sub-centers in Tabriz, a total of 13 and 14 health centers and sub-centers were chosen randomly using Randomizer software; and then in the selected centers and sub-centers, given their population, the proper size of each center was measured and determined with respect to the main sample size as proportional to the size for each center or sub-center. In the next stage of this process, all breastfeeding mothers covered by these centers were extracted and they were arranged with certain number and then were selected using Randomizer as much as the proportion was predicted for each center. The subjects were initially assessed in terms of the basic data and inclusion and exclusion criteria. In the case of failing to meet all inclusion criteria, or reluctance of the person to participate in the study or unavailability for any reason, the next woman was recruited randomly. They were called and were provided with some explanation about reasons and how the study will be conducted. Information about the study, its procedure and confidentiality were provided to eligible women and then they were invited to participate in the study. In the case of agreement, they were asked to complete the related questionnaires carefully and return them back to the researcher. The written informed consent was obtained from the participants.

\section{Data Collection Tool}

In this study, socio-demographic questionnaire, social support questionnaire (PRQ-85, Part 2: Personal Relationship Questionnaire-85), researcher-designed questionnaire of knowledge, Iowa Infant Feeding Attitude Scale (IIFAS), and Breastfeeding Self-efficacy Scale (BSES) were used to gather information. They all were filled out through self-completed method.

The socio-demographic questionnaire included items about age of mother and that of her husband, age of the infant, educational level, income, living with family, frequency of pregnancy, labor, infant's sex, and so forth.

The PRQ-85 was designed by Brandt and Weinert (36). It has 25 items which is ranked based on a Likert Scale from 1 to 7 (1=absolutely disagree, $2=$ disagree, $3=$ relatively disagree, $4=$ no idea, $5=$ relatively agree, $6=$ agree, and $7=$ absolutely agree) (36). The Cronbach $\alpha$ and ICC of this questionnaire were reported 0.84 and 0.9 , 
respectively $(37,38)$.

Breastfeeding self-efficacy questionnaire was designed by Deniss. This questionnaire is a 33-item scale and is scored by a 5-point Likert scale from totally agree (5) to totally disagree (1). Scores ranged from 33 to 165 . The score range of 33-76 was considered low self-efficacy, 77120 was average and 121-165 was regarded as high selfefficacy (13). The reliability of this questionnaire was reported $82 \%$ in the study by Varaei et al (1).

In this study, the attitude was assessed using IIFAS which was invented by Mora et al in 1998. The questionnaire includes 15 questions and is based on a Likert scale from 1 to 5 ( 1 =absolutely disagree, $2=$ disagree, $3=$ no idea, $4=$ agree, and $5=$ absolutely agree). Higher scores indicate a more positive attitude to breastfeeding and questions of $1,2,4,6,8,10$, and 13 were scored reversely. This questionnaire is a reliable and valid scale, and its Cronbach's alpha was reported between 0.85-0.86 (39).

In this study, knowledge was assessed using a researchermade questionnaire based on literature review and a combination of two questionnaires of Bahri et al (40) and Saeid et al (17). This questionnaire includes 14 questions which are assessed with three options: correct, incorrect and no idea. The correct answer was ranked with the score of 1 and incorrect and no idea options were ranked with score of zero and the scores varied from 0 to 14 .

Content and face validity were used for determining the validity of questionnaires and reliability was approved by intraclass correlation coefficient $(95 \% \mathrm{CI})$ which was 0.85 (0.68 to 0.93 ) for breastfeeding self-efficacy questionnaire, 0.98 (0.96 to 0.99 ) for PRQ, 0.89 (0.76 to 0.95$)$ for attitude questionnaire, and 0.71 (0.39 to 0.86$)$ for knowledge questionnaire.

\section{Data Analysis}

The collected data were statistically analyzed using SPSS (version 21.0). Descriptive statistics including frequency (percent) and mean (standard deviation) were used to describe socio-demographic characteristics, knowledge, attitude, social support, and breastfeeding self-efficacy. Pearson correlation test was used to determine the relationship between social support, knowledge, attitude and breastfeeding self-efficacy, and one-way analysis of variance (ANOVA) and independent $t$ test were used to determine the relationship between socio-demographic characteristics and breastfeeding self-efficacy. All variables which were related to breastfeeding self-efficacy based on bivariate tests (Pearson correlation, one-way ANOVA and independent $t$ tests) with $P<0.2$ were entered into the backward multivariate linear regression model.

\section{Results}

The socio-demographic characteristics of the participants are shown in Table 1. Participants' breastfeeding selfefficacy mean value (SD) was 138.6 (11.9) out of the achievable score of $33-165$. About $90 \%$ of the women had high and $10.3 \%$ of them had average breastfeeding selfefficacy.
Table 1. Socio-demographic Characteristics and Their Relationship With Breastfeeding Self-efficacy in Participants $(n=220)$

\begin{tabular}{|c|c|c|c|}
\hline Variable & Number & Mean (SD) & $P$ Value \\
\hline Age $(y)^{*}$ & & & 0.304 \\
\hline $15-25$ & 40 & $136.86(14.14)$ & \\
\hline $25-30$ & 93 & $138.18(11.93)$ & \\
\hline More than 30 & 85 & $140.13(10.69)$ & \\
\hline Education level & & & 0.450 \\
\hline Under diploma & 45 & $138.09(12.33)$ & \\
\hline Diploma & 94 & $138.82(11.52)$ & \\
\hline University degree & 81 & $140.01(12.23)$ & \\
\hline Occupation & & & 0.023 \\
\hline Housewife & 180 & $137.82(12.06)$ & \\
\hline Employee & 40 & $142.55(10.70)$ & \\
\hline Income & & & 0.891 \\
\hline Desirable & 32 & $138.69(11.08)$ & \\
\hline Fairly desirable & 138 & $138.43(11.76)$ & \\
\hline Undesirable & 50 & $139.38(13.14)$ & \\
\hline Number of pregnancy & & & 0.919 \\
\hline 1 & 89 & $138.55(12.11)$ & \\
\hline 2 & 103 & $138.58(11.47)$ & \\
\hline 3 and more & 28 & $139.95(8.54)$ & \\
\hline Infant age (month) & & & 0.219 \\
\hline 4 & 67 & $136.57(13.32)$ & \\
\hline 5 & 73 & $139.75(11.39)$ & \\
\hline 6 & 80 & $139.47(11.11)$ & \\
\hline Infant's gender & & & 0.697 \\
\hline Female & 102 & $138.34(12.27)$ & \\
\hline Male & 118 & $138.97(11.69)$ & \\
\hline Breastfeeding experience & & & 0.318 \\
\hline With experience & 109 & $139.49(11.79)$ & \\
\hline Without experience & 111 & $137.88(12.09)$ & \\
\hline Husband's age (y) & & & 0.124 \\
\hline $20-30$ & 56 & $135.14(13.00)$ & \\
\hline $30-35$ & 82 & $140.30(11.63)$ & \\
\hline More than 35 & 82 & $139.47(11.12)$ & \\
\hline Husband's education level & & & 0.184 \\
\hline Under diploma & 45 & $136.44(13.80)$ & \\
\hline Diploma & 92 & $138.24(11.69)$ & \\
\hline University degree & 83 & $140.38(11.01)$ & \\
\hline Living with family & & & 0.020 \\
\hline Independent & 168 & $139.72(11.39)$ & \\
\hline With husband/wife's family & 52 & $135.33(13.15)$ & \\
\hline Husband's occupation & & & 0.001 \\
\hline Unemployed/worker & 19 & $135.58(16.41)$ & \\
\hline Employee & 78 & $142.58(9.83)$ & \\
\hline Freelancer & 123 & $136.65(11.84)$ & \\
\hline Number of delivery & & & 0.677 \\
\hline 1 & 105 & $137.95(12.16)$ & \\
\hline 2 & 97 & $139.26(10.95)$ & \\
\hline 3 & 18 & $139.83(15.79)$ & \\
\hline Number of alive child & & & 0.665 \\
\hline 1 & 107 & $137.93(12.05)$ & \\
\hline 2 & 97 & $139.42(10.98)$ & \\
\hline 3 & 16 & $139.19(16.62)$ & \\
\hline Wanted pregnancy & & & 0.658 \\
\hline Yes & 193 & $138.80(11.93)$ & \\
\hline No & 27 & $137.69(12.41)$ & \\
\hline
\end{tabular}

*Two cases were without answers. 
Table 2. Breastfeeding Self-efficacy, Social Support, Self-efficacy, Knowledge and Attitude Conditions in Women With 4-6-month Babies ( $n=220$ )

\begin{tabular}{|c|c|c|c|c|c|}
\hline \multirow{2}{*}{ Variable } & \multirow{2}{*}{ Mean (SD) } & \multirow{2}{*}{ Achievable Score } & \multirow{2}{*}{ Achieved Score } & \multicolumn{2}{|c|}{ The relationship With Breastfeeding Self-efficacy } \\
\hline & & & & ( $P$ Value) & $r$ \\
\hline Breastfeeding self-efficacy & $138.6(11.9)$ & $33-165$ & $33-165$ & - & - \\
\hline Social support & $141.2(19.6)$ & $25-175$ & $52-175$ & $<0.001$ & 0.31 \\
\hline Knowledge & $10.3(2.3)$ & $0-14$ & $2-14$ & $<0.001$ & 0.36 \\
\hline Attitude & $61.0(6.6)$ & $15-75$ & $38-75$ & $<0.001$ & 0.35 \\
\hline
\end{tabular}

Abbreviation: SD, Standard deviation

The mean (SD) of social support score was measured 141.2 (19.6) out of 25-175 range and there was a significant statistical relationship between social support and breastfeeding self-efficacy $(P<0.001, r=0.31)$. The mean value $(\mathrm{SD})$ of knowledge and attitude scores were 10.3 (2.3) and 61.0 (6.6), respectively, out of 0 -14 and 1575. There was a significant positive correlation between breastfeeding self-efficacy and knowledge $(P<0.001$, $\mathrm{r}=0.36)$ and attitude $(P<0.001, \mathrm{r}=0.35)$ (Table 2).

According to bivariate tests, the variables of social support, knowledge, attitude, husband's age, mother's occupation, husband's occupation and education, and living with family that had $P<0.2$ entered into backward multiple linear regression model for controlling the confounding variables. The variables of mother's job, husband's job, his education and living with family were excluded from the model and variables of social support, knowledge, attitude and husband's age remained in the model and explained $19.2 \%$ of variance in breastfeeding self-efficacy score (Table 3 ).

\section{Discussion}

In this study, the mean score of breastfeeding in the mothers was high and most mothers had high breastfeeding selfefficacy. Social support, knowledge, attitude and husband's age were the predictors of breastfeeding self-efficacy in the mothers by modifying other socio-demographic variables.

In this study, the mean score of breastfeeding selfefficacy was 138.6 and most participants had high breastfeeding self-efficacy. The results of this study were compatible with the results of the study by Varaei et al on the breastfeeding women in Tehran (1); however this mean

Table 3. The Predictors of Breastfeeding Self-efficacy in Breastfeeding Mothers With 4-6-month Babies According to Linear Regression Model $(n=220)$

\begin{tabular}{lll}
\hline Variable & B (95\% CI ) & $P$ \\
\hline Social support & $0.10(0.02$ to 0.18$)$ & 0.016 \\
Knowledge & $1.18(0.46$ to 1.89$)$ & 0.001 \\
Attitude & $0.32(0.07$ to 0.58$)$ & 0.013 \\
Husband's age (Reference: above 35$)$ & & \\
Under 30 & $-2.48(-6.19$ to 1.23$)$ & 0.189 \\
30-35 & $0.90(-2.41$ to 4.21$)$ & 0.593 \\
\hline
\end{tabular}

Adjusted $\mathrm{R}^{2}: 19.2 \%$. score was higher than the mean score of self-efficacy in the breastfeeding mothers in the study by Hasanpoor et al in Ahwaz (23). Delivery and breastfeeding experiences can affect breastfeeding self-efficacy. The reason for difference between the results of the present study and those of the study by Hasanpoor et al is probably that in their study, not all the participants had breastfeeding experience while in this study, almost half of the participants had already breastfeeding experience.

There was a significant statistical relationship between breastfeeding self-efficacy and social support in this study. In the study by Dodgson et al about socio-cultural patterns related to breastfeeding, the results showed that breastfeeding is affected by cultural patterns including current culture, socio-economic status and social support (41). The results of a review by Meedya et al showed that husband's attitude toward breastfeeding, interventions like breastfeeding training and husbands' and parents' support affect breastfeeding (35).

In a qualitative study, Nesbitt et al focused on facilitating and inhibiting factors of breastfeeding on 16 Canadian women (27). The results showed that family and husband's attitudes affect breastfeeding and altogether factors like friendly and social relations, social support, mother's knowledge and her feel of convenience promote breastfeeding. The results of these studies are compatible with the results of present study, however, the results of the study by McCarter-Spaulding and Gore in America on 155 breastfeeding women of African descent showed that there was not a direct relationship between social support and duration and pattern of breastfeeding. This result is not compatible with the results of this study (42). The reason may be cultural differences.

Perceived social support increases self-care and selfconfidence and has positive effect on the individual's physical, psychological and social conditions and promotes performance (43). The results of the study by O'Campo et al showed that the women with low breastfeeding self-efficacy are 3.1 times more than the women with high breastfeeding self-efficacy on the verge of stopping exclusive breastfeeding (16). There is a significant relationship between the increase of breastfeeding selfefficacy and the increase of breastfeeding duration (13), so high breastfeeding self-efficacy in mothers is important.

Regarding the effective role of social support, mother's knowledge and attitude in increasing breastfeeding, 
cultural variables and social support should be focused beside socio-demographic variables in designing the interventions for breastfeeding promotion. Moreover, mother's knowledge should be promoted and their attitude toward breastfeeding should be modified.

One of the limitations of the present study is its crosssectional nature. The relationship between self-efficacy and socio-demographic variables do not necessarily represent cause and effect relation. In addition, as this study was conducted on 220 breastfeeding mothers having 4-6-month infants, another limitation of this study was impossibility of surveying breastfeeding selfefficacy along with duration of breastfeeding. The results of this study can be used in family training, supporting breastfeeding mothers by family and society, promoting mother's knowledge and modifying their attitude in order to consequently improve breastfeeding practice in the mothers.

\section{Conclusions}

Considering the relationship between breastfeeding selfefficacy and social support, knowledge and attitude, breastfeeding self-efficacy can be promoted by informing the mothers, modifying their attitude and increase of family and society support from the mothers.

\section{Conflict of interests}

The authors declare no conflict of interests.

\section{Financial Support}

This study was supported by the Research Deputy of Tabriz University of Medical Sciences.

\section{Ethical Issues}

The Ethics Committee of Tabriz University of Medical Sciences approved the study (code of ethics: 5/4/7619).

\section{Acknowledgements}

Hereby, cooperation and assistance of the health centers of Tabriz and all women who participated in this study are appreciated.

\section{References}

1. Varaei S, Mehrdad N, Bahrani N. The Relationship between Self-efficacy and Breastfeeding, Tehran, Iran. Hayat. 2009;15(3):31-38.

2. Beral V, Bull D, Doll R, Peto R, Reeves G. Breast cancer and abortion: collaborative reanalysis of data from 53 epidemiologicalstudies, including 83?000 women with breast cancer from 16 countries. Lancet. 2004;363(9414):10071016. doi:10.1016/s0140-6736(04)15835-2

3. Britton C, McCormick FM, Renfrew MJ, Wade A, King SE. Support for breastfeeding mothers. Cochrane Database Syst Rev. 2007(1):Cd001141. doi:10.1002/14651858.CD001141. pub3

4. Kramer MS, Chalmers B, Hodnett ED, et al. Promotion of Breastfeeding Intervention Trial (PROBIT): a randomized trial in the Republic of Belarus. JAMA. 2001;285(4):413420 .
5. Lucas A, Brooke OG, Morley R, Cole TJ, Bamford MF. Early diet of preterm infants and development of allergic or atopic disease: randomised prospective study. BMJ. 1990;300(6728):837-840.

6. Pollard D, Cuill M, Wilmigton N. The Relationship between Baseline Self-Efficacy and Breastfeeding duration. South Online J Nurs Res. 2009;9(4).

7. Sadauskaite-Kuehne V, Ludvigsson J, Padaiga Z, Jasinskiene E, Samuelsson U. Longer breastfeeding is an independent protective factor against development of type 1 diabetes mellitus in childhood. Diabetes Metab Res Rev. 2004;20(2):150-157. doi:10.1002/dmrr.425

8. Singhal A, Cole TJ, Lucas A. Early nutrition in preterm infants and later blood pressure: two cohorts after randomised trials. Lancet. 2001;357(9254):413-419. doi:10.1016/s0140-6736(00)04004-6

9. World Health Organization. Global health risks: mortality and burden of disease attributable to selected major risks. Geneva: World Health Organization; 2009.

10. Shears E. Meeting the iron needs of infants and young children: an update. CMAJ. 1991;145(12):1558-1559.

11. Almasi H, Saberi H, Moravveji SA. The pattern of exclusive breast feeding in neonates under healthcares in health centers of Kashan city during 2006. Feyz. 2010;14(2):163168.

12. Rashidian A, Karimi-Shahanjarini A, Khosravi A, et al. Iran's Multiple Indicator Demographic and Health Survey - 2010: Study Protocol. Int J Prev Med. 2014;5(5):632-642.

13. Dennis CL. The breastfeeding self-efficacy scale: psychometric assessment of the short form. J Obstet Gynecol Neonatal Nurs. 2003;32(6):734-744.

14. Blyth R, Creedy DK, Dennis CL, Moyle W, Pratt J, De Vries SM. Effect of maternal confidence on breastfeeding duration: an application of breastfeeding self-efficacy theory. Birth. 2002;29(4):278-284.

15. Gijsbers B, Mesters I, Knottnerus JA, van Schayck CP. Factors associated with the duration of exclusive breast-feeding in asthmatic families. Health Educ Res. 2008;23(1):158-169. doi:10.1093/her/cym013

16. O'Campo P, Faden RR, Gielen AC, Wang MC. Prenatal factors associated with breastfeeding duration: recommendations for prenatal interventions. Birth. 1992;19(4):195-201. doi:10.1111/j.1523-536X.1992.tb00402.x

17. Saied H, Mohamed A, Suliman A, Al Anazi W. Breastfeeding knowledge, Attitude and Barriers among Saudi Women in Riyadh. J Nat Sci Res. 2013;3(12):6-13.

18. Taveras EM, Capra AM, Braveman PA, Jensvold NG, Escobar GJ, Lieu TA. Clinician support and psychosocial risk factors associated with breastfeeding discontinuation. Pediatrics. 2003;112(1 Pt 1):108-115.

19. O’Brien M, Buikstra E, Hegney D. The influence of psychological factors on breastfeeding duration. J Adv Nurs. 2008;63(4):397-408. doi:10.1111/j.1365-2648.2008.04722.x

20. Schwartz K, D’Arcy HJ, Gillespie B, Bobo J, Longeway M, Foxman B. Factors associated with weaning in the first 3 months postpartum. J Fam Pract. 2002;51(5):439-444.

21. Handayani L, Kosnin AM, Jiar YK. Social support, knowledge, attitude, and self-efficacy as predictors on breastfeeding practice. https://pdfs.semanticscholar. org/e4de/fc3b1f9bc7fa1fb71a32d4be917b276826c3.pdf. Published 11 Sep 2017.

22. Rahmatnejad L, Bastani F. An investigation of breast feeding self efficacy and its relationship with exclusive 
breast feeding. J Alborz Health. 2012;1(1):31-6.

23. Hasanpour S, Bani S, Ansari S, Ebrahimi H. Measuring breastfeeding self - efficacy among pregnant women referred to health centers of Ahvaz. Nursing and Midwifery Journal. 2011;5(19):47-53.

24. Baheiraei A, Mirghafourvand M, Charandabi SM, Mohammadi E, Nedjat S. Health-promoting behaviors and social support in Iranian women of reproductive age: a sequential explanatory mixed methods study. Int J Public Health. 2014;59(3):465-473. doi:10.1007/s00038-0130513-y

25. Barona-Vilar C, Escriba-Aguir V, Ferrero-Gandia R. A qualitative approach to social support and breast-feeding decisions. Midwifery. 2009;25(2):187-194. doi:10.1016/j. midw.2007.01.013

26. Ericson J, Eriksson M, Hellstrom-Westas L, Hagberg L, Hoddinott P, Flacking R. The effectiveness of proactive telephone support provided to breastfeeding mothers of preterm infants: study protocol for a randomized controlled trial. BMC Pediatr. 2013;13:73. doi:10.1186/1471-2431-1373

27. Nesbitt SA, Campbell KA, Jack SM, Robinson H, Piehl K, Bogdan JC. Canadian adolescent mothers' perceptions of influences on breastfeeding decisions: a qualitative descriptive study. BMC Pregnancy Childbirth. 2012;12:149. doi:10.1186/1471-2393-12-149

28. Gill SL, Reifsnider E, Mann AR, Villarreal P, Tinkle MB. Assessing infant breastfeeding beliefs among low-income mexican americans. J Perinat Educ. 2004;13(3):39-50. doi:10.1624/105812404x1761

29. Mosalli R, Abd El-Azim AA, Qutub MA, Zagoot E, Janish $\mathrm{M}$, Paes BA. Perceived barriers to the implementation of a baby friendly initiative in Jeddah, Saudi Arabia. Saudi Med J. 2012;33(8):895-900.

30. Dadhich JP, Agarwal RK. Mainstreaming early and exclusive breastfeeding for improving child survival. Indian Pediatr. 2009;46(1):11-17.

31. Issler H, de Sa MB, Senna DM. Knowledge of newborn health care among pregnant women: basis for promotional and educational programs on breastfeeding. Sao Paulo Med J. 2001;119(1):7-9.

32. Serenius F, Swailem AR, Edressee AW, Hofvander Y. Patterns of breastfeeding and weaning in Saudi Arabia.
Acta Paediatr Scand Suppl. 1988;346:121-129.

33. Piper S, Parks PL. Predicting the duration of lactation: evidence from a national survey. Birth. 1996;23(1):7-12.

34. Uchendu UO, Ikefuna AN, Emodi IJ. Exclusive breastfeeding--the relationship between maternal perception and practice. Niger J Clin Pract. 2009;12(4):403406.

35. Meedya S, Fahy K, Kable A. Factors that positively influence breastfeeding duration to 6 months: a literature review. Women Birth. 2010;23(4):135-145. doi:10.1016/j. wombi.2010.02.002

36. Weinert C, Brandt PA. Measuring social support with the Personal Resource Questionnaire. West J Nurs Res. 1987;9(4):589-602. doi:10.1177/019394598700900411

37. Baheiraei A, Mirghafourvand M, Mohammadi E, Charandabi SM, Nedjat S. Social support for women of reproductive age and its predictors: a population-based study. BMC Womens Health. 2012;12:30. doi:10.1186/14726874-12-30

38. Mirghafourvand M, Baheiraei A, Nedjat S, Mohammadi E, Charandabi SM, Majdzadeh R. A population-based study of health-promoting behaviors and their predictors in Iranian women of reproductive age. Health Promot Int. 2015;30(3):586-594. doi:10.1093/heapro/dat086

39. de la Mora A, Russell DW, Dungy CI, Losch M, Dusdieker L. The Iowa Infant Feeding Attitude Scale: Analysis of Reliability and Validity. J Appl Soc Psychol. 1999;29(11):2362-2380.

40. Bagheri S, Bahri N, Rahmani R, Erfan M, Tolidi H. The comparison of workshop training and offering booklet on knowledge, health beliefs and breastfeeding behavior after delivery. Iran J Obstet Gynecol Infertil. 2013;15(32):14-22.

41. Dodgson JE, Duckett L, Garwick A, Graham BL. An ecological perspective of breastfeeding in an indigenous community. J Nurs Scholarsh. 2002;34(3):235-241.

42. McCarter-Spaulding D, Gore R. Breastfeeding selfefficacy in women of African descent. J Obstet Gynecol Neonatal Nurs. 2009;38(2):230-243. doi:10.1111/j.15526909.2009.01011.x

43. Baheiraei A, Mirghafourvand M, Charandabi SM, Mohammadi E. Facilitators and Inhibitors of Healthpromoting Behaviors: The Experience of Iranian Women of Reproductive Age. Int J Prev Med. 2013;4(8):929-939.

(c) 2018 The Author (s); This is an open-access article distributed under the terms of the Creative Commons Attribution License (http://creativecommons.org/licenses/by/4.0), which permits unrestricted use, distribution, and reproduction in any medium, provided the original work is properly cited. 